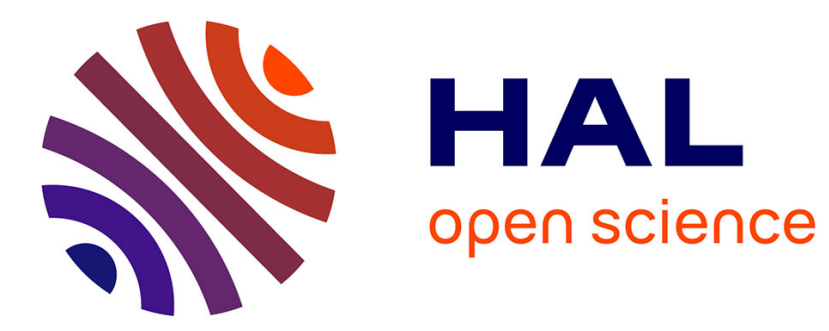

\title{
Towards general measures of comparison of objects
}

Bernadette Bouchon-Meunier, Maria Rifqi, S. Bothorel

\section{To cite this version:}

Bernadette Bouchon-Meunier, Maria Rifqi, S. Bothorel. Towards general measures of comparison of objects. Fuzzy Sets and Systems, 1996, 84 (2), pp.143-153. hal-01072162

\section{HAL Id: hal-01072162 \\ https://hal.inria.fr/hal-01072162}

Submitted on 7 Oct 2014

HAL is a multi-disciplinary open access archive for the deposit and dissemination of scientific research documents, whether they are published or not. The documents may come from teaching and research institutions in France or abroad, or from public or private research centers.
L'archive ouverte pluridisciplinaire HAL, est destinée au dépôt et à la diffusion de documents scientifiques de niveau recherche, publiés ou non, émanant des établissements d'enseignement et de recherche français ou étrangers, des laboratoires publics ou privés. 


\title{
Towards general measures of comparison of objects
}

\author{
Bernadette Bouchon-Meunier ${ }^{\mathrm{a}, *}$, Maria Rifqi $^{\mathrm{a}}$, Sylvie Bothorel $^{\mathrm{a}, \mathrm{b}}$ \\ ${ }^{a}$ LAFORIA-IBP, Université Pierre et Marie Curie, Case 169, 4 place Jussieu, 75252 Paris Cedex 05, France \\ ${ }^{\mathrm{b}}$ General Electric Medical Systems, 283, rue de la Minière, B.P. 34, 78533 Buc, France
}

Received February 1996

\begin{abstract}
We propose a classification of measures enabling to compare fuzzy characterizations of objects, according to their properties and the purpose of their utilization. We establish the difference between measures of satisfiability, resemblance, inclusion and dissimilarity. We base our study on concepts analogous to those developed by A. Tversky for his general work on similarities.
\end{abstract}

Keywords: Comparison of objects; Satisfiability; Resemblance; Similarity; Aggregation

\section{Introduction}

The comparison of descriptions of objects is a usual operation in many domains: psychology, analogy, physical sciences, image processing, clustering, deductive reasoning, case-based reasoning. This comparison is frequently achieved through a measure intended to determine to which extent the descriptions have common points or differ from each other. The measures of comparison have various forms $[30,8,6]$, depending on the purpose of their utilization. In this paper, we address four kinds of measures of comparison: measures of satisfiability, resemblance and inclusion which can be regarded as measures of similitude, and measures of dissimilarity. It is, for instance, possible to use measures of similitude in deductive reasoning to evaluate to which extent an observation satisfies a given rule or in case-based reasoning to measure the resemblance between the characteristics of a known case and a new one.

\footnotetext{
*Corresponding author. E-mail: bouchon@laforia.ibp.fr.
}

The satisfiability corresponds to a situation in which we consider a reference object or class and we decide if a new object is compatible with it or satisfies the reference. This situation is typical in prototype-based reasoning, where the references are prototypes and a new object must be associated with one of them.

The inclusion also concerns a situation with a reference object and measures if the points common to $A$ and $B$ are important with regard to $A$. It can be used in database management systems to decide if a class is included in another one [19, 14].

The resemblance is used for a comparison between the descriptions of two objects, of the same level of generality, to decide if they have many common characteristics. This situation occurs naturally in a case-based reasoning system. It is also the basis of similarity logic [20,9].

The dissimilarity between objects evaluates to which extent they are different. This quantity may be useful when, in the retrieval step of a case-based reasoning system, no case is sufficiently similar to the new case. It is then interesting to be able to establish 
comparison with respect to differences between descriptions, and to choose the least different case from the new one.

Many measures of comparison have been proposed and studied, generally in a given framework of application. We propose a general form for measures of comparison, enabling us to classify the main existing ones with respect to their properties and utilizations, and to suggest new quantities.

\section{Comparison of fuzzy sets}

We consider objects with imperfect descriptions, affected with imprecisions and inaccuracies. We use the framework of fuzzy sets to represent such descriptions. Crisp sets representing precise and certain descriptions of objects will be regarded as particular cases of fuzzy sets.

\subsection{Basic notions}

For any set $\Omega$ of elements, let $F(\Omega)$ denote the set of fuzzy subsets of $\Omega, f_{A}$ the membership function of any description $A$ in $F(\Omega)$ and $\operatorname{supp}(A)=\{x \in$ $\left.\Omega \mid f_{A}(x) \neq 0\right\}$ its support. The comparison of two fuzzy sets $A$ and $B$ defined on a given universe takes into account the elements of the universe which belong, at least partly, to each of them. Depending on the situation, we prefer to consider the elements which belong to $A$ and not to $B$, to $B$ and not to $A$, or to both of them. We take also into account the degree of membership of these elements to $A$ and $B$ and, finally, the weight of the part of the universe common to $A$ and $B$ or relevant to only one of them. We use the classical definition of intersection: $f_{A \cap B}=\min \left(f_{A}, f_{B}\right)$ to describe the elements belonging to $A$ and $B$. We suppose that we are given a means of evaluating the weight of the elements of the universe [10] characterized by a fuzzy set through a fuzzy set measure.

Definition 2.1. A fuzzy set measure $M$ is a mapping: $F(\Omega) \rightarrow \mathbb{R}^{+}$such that, for every $A$ and $B$ in $F(\Omega)$ :

$\mathrm{AM} 1: M(\emptyset)=0$,

AM2: if $B \subseteq A$, then $M(B) \leqslant M(A)$.

If the values of $M$ are restricted to $[0,1], M$ is a fuzzy set measure.
The following examples of fuzzy set measures will be used in measures of comparison:

$$
\begin{aligned}
M_{1}(A) & =\int_{\Omega} f_{A}(x) \mathrm{d} x, \\
M_{2}(A) & =\sup _{x \in \Omega} f_{A}(x), \\
M_{3}(A) & =\sum_{\text {count }}(A) \\
& =\sum_{x \in \Omega} f_{A}(x) \quad \text { if } \Omega \text { is finite [5], }
\end{aligned}
$$

$M_{4}(A)=|\operatorname{Supp}(A)|$ if $|$.$| denotes a metrics on \Omega$,

$$
M_{5}(A)=\sum_{\Omega} f_{A}(x)^{r} \text { if } \Omega \text { is finite and } r \text { is an integer. }
$$

A fuzzy set measure enables us to evaluate the weight of the part of the universe common to $A$ and $B$ if we consider $M(A \cap B)$, for instance. The identification of elements of the universe which belong to $A$ and not to $B$, or conversely, is achieved by means of an operation called difference.

Definition 2.2. An operation on $F(\Omega)$ is called a difference and denoted by - , if it satisfies for every $A$ and $B$ in $F(\Omega)$ :

D1: if $A \subseteq B$, then $A-B=\emptyset$,

D2: $B-A$ is monotonous with regard to $B: B \subseteq B^{\prime}$ entails $B-A \subseteq B^{\prime}-A$.

This definition is slightly different from the definition of difference given by Kaufmann [11], or by Roberts [18] or also Dubois and Prade [7], but it is compatible with the definition of a difference in the case of crisp subsets of $\Omega$, where $A-B$ can be defined as the complement of $A \cap B$ in $A$ or, equivalently to the intersection of $A$ with the complement of $B$ in $\Omega$.

Examples of differences are the following:

$$
\begin{aligned}
& f_{A-1 B}(x)=\max \left(0, f_{A}(x)-f_{B}(x)\right) \quad[29], \\
& f_{A-{ }_{2} B}(x)= \begin{cases}f_{A}(x) & \text { if } f_{B}(x)=0, \\
0 & \text { if } f_{B}(x)>0 .\end{cases}
\end{aligned}
$$




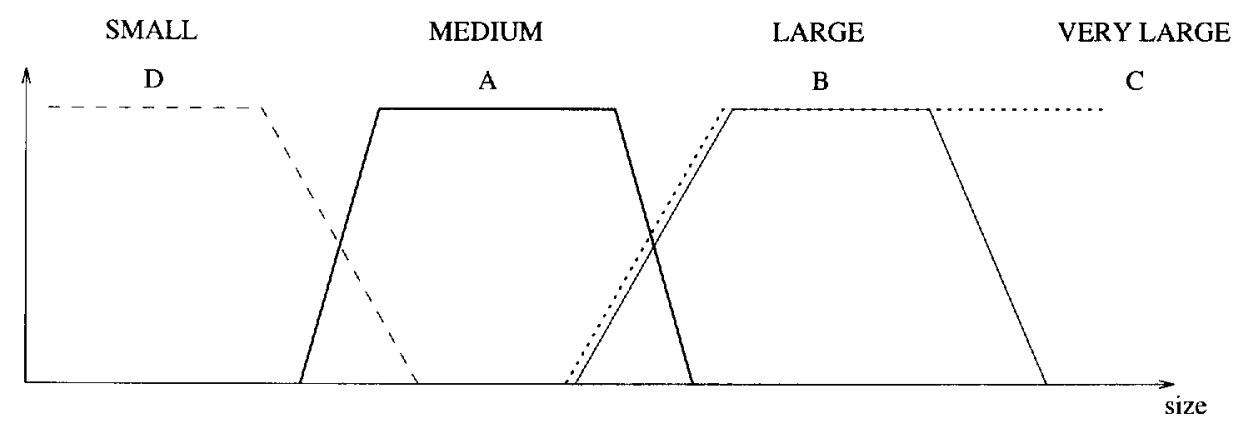

Fig. 1. Difference between a measure of comparison and an $M$-measure of comparison.

\subsection{Measure of comparison}

The simplest form of measure of comparison between $A$ and $B$ takes into account $A \cap B, B-A$ and $A-B$

Definition 2.3. A measure of comparison on $\Omega$ is a mapping $S: F(\Omega) \times F(\Omega) \rightarrow[0,1]$ such that $S(A, B)$ $=G_{S}(A \cap B, B-A, A-B)$, for a given mapping $G_{S}: F(\Omega) \times F(\Omega) \times F(\Omega) \rightarrow[0,1]$.

Depending on the properties of $G_{S}$, we obtain various measures of the proximity of two fuzzy sets. Since the order on $F(\Omega)$ induced by the inclusion of fuzzy sets is not total, a measure of similitude is insufficient to compare any pair of fuzzy sets $A$ and $B$. Further, it can be useful to compare two fuzzy sets $A$ and $B$ more globally than by means of this order only. We introduce a less restrictive measure of comparison.

Definition 2.4. An $M$-measure of comparison on $\Omega$ is a mapping $S: F(\Omega) \times F(\Omega) \rightarrow[0,1]$ such that $S(A, B)=F_{S}(M(A \cap B), M(B-A), M(A-B))$, for a given mapping $F_{S}: \mathbb{R}^{+} \times \mathbb{R}^{+} \times \mathbb{R}^{+} \rightarrow[0,1]$ and a fuzzy measure $M$ on $\Omega$.

We can see the difference between a measure of comparison and an $M$-measure of comparison on the example given in Fig. 1, where: $A \cap B=A \cap C$ and $A-{ }_{1} B=A-{ }_{1} C, M_{1}(A \cap D)=M_{1}(A \cap B)=$ $M_{1}(A \cap C), M_{1}\left(A-{ }_{1} D\right)=M_{1}\left(A-{ }_{1} B\right)$ but $A \cap D \neq$ $A \cap B$ and $A-{ }_{1} D \neq A-{ }_{1} B$.

It is obvious that any measure of comparison is an $M$-measure of comparison. For instance, we can consider only crisp subsets of $\Omega$ and $M_{1}, M_{3}, M_{4}$ or $M_{5}$ are equivalent.
The same kind of question has been addressed by Tversky who has given an axiomatic theory of similarity [25] on a set of features $\Omega$. It is compatible with our definition of a measure of comparison since he defines the similarity of $B$ to $A$ as: $S(A, B)=f(A \cap B, B-A, A-B)$ (Example 1). The only difference lies in the fact that his measures take values in $\mathbb{R}^{+}$instead of $[0,1]$. Implicitly, $f$ takes into account a kind of measure of subsets, since he considers that $V \simeq W$ if they are the intersection or the difference between two features providing the same value of similarity. In that, we can consider that he uses $M$-measures of comparison for a given measure $M$, for instance, $M_{3}$. He further proves [23] that $f$ is necessarily increasing in $B-A$ and $A-B$, which provides a particular class of $M$-measures of comparison, which we shall address in the next section.

In this paper, we shall restrict ourselves to the study of $M$-measures of comparison.

\section{Similitude of fuzzy sets}

For two fuzzy subsets $A$ and $B$ of $\Omega$, either we want to have a look at the resemblance between them or we are interested in their differences. First of all, let us focus on the first case.

Definition 3.1. An $M$-measure of similitude on $\Omega$ is an $M$-measure of comparison $S$ satisfying

AF1: $F_{S}(u, v, w)$ is non-decreasing in $u$, nonincreasing in $v$ and $w$.

This definition is justified by the following proposition which corresponds to natural requirements for 
a quantity evaluating to which extent $A$ and $B$ are similar.

Proposition 3.1. An $M$-measure of similitude $S$ satisfies the following properties:

P1: $M$-monotonicity. If $M(A \cap B) \geqslant M\left(A \cap B^{\prime}\right)$ and $M(B-A) \leqslant M\left(B^{\prime}-A\right)$ and $M(A-B) \leqslant M\left(A-B^{\prime}\right)$, then $S(A, B) \geqslant S\left(A, B^{\prime}\right)$.

P2: Monotonicity. If $A \cap B \supseteq A \cap B^{\prime}$ and $B-A \subseteq$ $B^{\prime}-A$ and $A-B \subseteq A-B^{\prime}$, then $S(A, B) \geqslant S\left(A, B^{\prime}\right)$.

Obviously, P1 and P2 are consequences of AF1. The "if" part of P2 is a particular case of the "if" part of P1. Their interest lies in the fact that this property of monotonicity is coherent with the monotonicity of the contrast model given by Tversky [25].

The following properties are also direct consequences of axiom AF1; we mention them because they express the influence of the three elements $M(A \cap B), M(A-B)$ and $M(B-A)$ in a clearer way which can be visualized in Fig. 1, for instance.

Proposition 3.2. An $M$-measure of similitude $S$ satisfies the following additional properties:

P3: $M$-elementary left internal information. If $M(A \cap B)=M\left(A \cap B^{\prime}\right)$ and $M(A-B)=M\left(A-B^{\prime}\right)$, then $M(B-A) \leqslant M\left(B^{\prime}-A\right)$ entails $S(A, B) \geqslant$ $S\left(A, B^{\prime}\right)$.

P4: $M$-elementary right internal information. If $M(A \cap B)=M\left(A \cap B^{\prime}\right)$ and $M(B-A)=$ $M\left(B^{\prime}-A\right)$, then $M(A-B) \leqslant M\left(A-B^{\prime}\right)$ entails $S(A, B) \geqslant S\left(A, B^{\prime}\right)$.

P5: $M$-elementary external information. If $M(A-B)=M\left(A-B^{\prime}\right)$ and $M(B-A)=M\left(B^{\prime}-A\right)$, then $M(A \cap B) \geqslant M\left(A \cap B^{\prime}\right)$ entails $S(A, B) \geqslant$ $S\left(A, B^{\prime}\right)$.

These three properties can be interpreted as follows:

- For a given amount of information common to the descriptions $A$ and $B$ and a given amount of information contained in $A$ and not in $B$, the smaller the information contained in $B$ and not in $A$, the more $A$ and $B$ are alike.

- For a given amount of information contained in $A$ and not in $B$ and a given amount of information contained in $B$ and not in $A$, the smaller the information common to the descriptions $A$ and $B$, the more $A$ and $B$ are alike.
The relation given by Tversky [25]: $S(A, B)=$ $f(A \cap B) /(f(A \cap B)+\alpha f(A-B)+\beta f(B-A))$ $\alpha, \beta \geqslant 0$ (Example 2) is an $f$-measure of similitude if $f$ is a fuzzy set measure.

Another very particular case of $M$-measure of similitude is the well-known quantity (the possibility of $B$ with respect to $A$ ) introduced by Dubois and Prade [8]: $\quad S(A, B)=\sup _{x} \min \left(f_{A}(x), f_{B}(x)\right)$ (Example 3) for normalized fuzzy sets, with the fuzzy set measure $M_{2}$, whatever the difference may be.

Measures of similitude are still very general and we can identify three important classes of such measures. In the two first ones, $A$ is considered as a reference and $B$ is compared to $A$. Thus, these measures are not symmetrical. In the third one, $A$ and $B$ have the same kind of status and none of them is a reference. Thus, this measure is symmetrical.

\subsection{Measures of satisfiability}

The first family of measures of similitude we consider evaluates the satisfiability of a reference description $A$ of $F(\Omega)$ by a new description $B$ defined as a fuzzy subset of $\Omega$ [3].

Definition 3.2. An $M$-measure of satisfiability on $\Omega$ is an $M$-measure of similitude $S$ such that:

$\mathrm{AF} 2: F_{S}(0, v, w)=0$ whatever $v$ and $w$ may be,

AF3: $F_{S}(u, v, w)$ is independent of $w$,

AF4: $F_{S}(u, 0,)=$.1 whatever $u \neq 0$ may be.

Properties given in Proposition 3.1 are still valid for $M$-measures of satisfiability which also satisfy additional properties taking into account their specificity. Most of them are extended versions of the properties required by Tversky from his notion of similarity.

Proposition 3.3. Based on the order of descriptions deduced from the inclusion of fuzzy sets, the following properties hold for an $M$-measure of satisfiability:

P6: Exclusiveness. If $A \cap B=\emptyset$ then $S(A, B)=$ $S(B, A)=0$.

P7: Containment. If $B \subseteq A, B \neq \emptyset$, then $S(A, B)$ $=1$.

P8: Independence. If $A \cap B=C \cap D, A^{\prime} \cap B^{\prime}=$ $C^{\prime} \cap D^{\prime}, B-A=B^{\prime}-A^{\prime}$ and $D-C=D^{\prime}-C^{\prime}$, then $S(A, B) \geqslant S\left(A^{\prime}, B^{\prime}\right)$ if and only if $S(C, D) \geqslant S\left(C^{\prime}, D^{\prime}\right)$. 
P9: Relative independence. If $A \cap B=A \cap D$, $A \cap B^{\prime}=A \cap D^{\prime}, B-A=B^{\prime}-A$ and $D-A=D^{\prime}-A$, then $S(A, B) \geqslant S\left(A, B^{\prime}\right)$ if and only if $S(A, D)$ $\geqslant S\left(A, D^{\prime}\right)$

Proof.

- Obviously, P6 is a direct consequence of AF2.

- P7 is a consequence of AF4.

- Let us prove property P8.

$$
\begin{aligned}
S(A, B) \geqslant S\left(A^{\prime}, B^{\prime}\right) \Leftrightarrow & F_{S}(M(A \cap B), M(B-A)) \\
& \geqslant F_{S}\left(M\left(A^{\prime} \cap B^{\prime}\right), M\left(B^{\prime}-A^{\prime}\right)\right) \\
\Leftrightarrow & F_{S}\left(M(C \cap D), M\left(B^{\prime}-A^{\prime}\right)\right) \\
& \geqslant F_{S}\left(M\left(C^{\prime} \cap D^{\prime}\right), M\left(B^{\prime}-A^{\prime}\right)\right) \\
\Leftrightarrow & \left.M(C \cap D) \geqslant M\left(C^{\prime} \cap D^{\prime}\right)\right),
\end{aligned}
$$

further $M(D-C)=M\left(D^{\prime}-C^{\prime}\right)$; hence, because of $\mathrm{AF} 1, S(C, D) \geqslant S\left(C^{\prime}, D^{\prime}\right)$

- P9 is easily inferred from P8.

The independence property can be interpreted as follows: it is equivalent to compare the resemblance of a first pair $(A, B)$ to the resemblance of a second pair $\left(A^{\prime}, B^{\prime}\right)$ with the same difference, or to compare the resemblance of any other first pair $(C, D)$ to the resemblance of any other second one $\left(C^{\prime}, D^{\prime}\right)$, also with the same difference, as soon as the information common to the first pair is preserved, the information common to the second pair is preserved.

The relative independence is a particular case of the independence, in the case where $C, A^{\prime}, C^{\prime}$ are replaced by $A$. It means that, for a given reference object $A$, it is equivalent to compare the resemblance of any object $B$ with it to the resemblance of any object $B^{\prime}$ having the same difference with $A$, for a given information common to $A$ and $B$, to $A$ and $B^{\prime}$.

We notice that the model given by Tversky as $S(A, B)=f(A \cap B, B-A, A-B)$ can be regarded as an $M$-measure of satisfiability $S$, provided that $f(u, v, w)$ is independent of $w$ and, again, increasing in $u$ and decreasing in $v$. The properties of independence and relative independence of $S$ are compatible with the independence defined by Tversky. For instance, our second example (Example 2) with $\alpha=0$ is an $M$ measure of satisfiability if $f$ is a fuzzy set measure.

Obviously, the property of containment entails a reflexivity property: $S(A, A)=1$.
Proposition 3.4. An $M$-measure of satisfiability $S$ has the following properties:

P10: Exemplarity. If $M(B-A) \geqslant M(A-B)$, then $S(A, B) \leqslant S(B, A)$.

P11: $M$-elementary internal information. If $M(A \cap B)=M\left(A \cap B^{\prime}\right)$, then $M(B-A) \leqslant M\left(B^{\prime}-A\right)$ entails $S(A, B) \geqslant S\left(A, B^{\prime}\right)$.

P12: $M$-elementary external information. If $M(B-A)=M\left(B^{\prime}-A\right)$, then $M(A \cap B) \geqslant M\left(A \cap B^{\prime}\right)$ entails $S(A, B) \geqslant S\left(A, B^{\prime}\right)$.

P13: Solvability. For any $A, B, B^{\prime}$ such that $M(A \cap B)>M\left(A \cap B^{\prime}\right)$ and $M(B-A)<M\left(B^{\prime}-A\right)$, there exists a pair $(P, Q)$ such that $M(P \cap Q)=$ $M(A \cap B), M(Q-P)=M\left(B^{\prime}-A\right)$ and $S(A, B)>$ $S(P, Q)>S\left(A, B^{\prime}\right)$ if $F_{S}(u, v,$.$) is strictly increasing$ in $u$ and strictly decreasing in $v$.

\section{Proof.}

- Let us prove the exemplarity property. Assume that: $M(B-A) \geqslant M(A-B)$.

$$
\begin{aligned}
S(A, B) & =F_{S}(M(A \cap B), M(B-A)) \\
& \leqslant F_{S}(M(A \cap B), M(A-B)) \\
& =S(B, A) .
\end{aligned}
$$

- Properties P11 and P12 are simplified versions of properties $\mathrm{P} 3$ and $\mathrm{P} 5$, due to the independence of $F_{S}$ with regard to $w$.

- Let us prove the solvability property.

Assume that $M(A \cap B)>M\left(A \cap B^{\prime}\right)$ and $M(B-A)$ $<M\left(B^{\prime}-A\right)$. If there exist $P$ and $Q$ such that $M(P \cap Q)=M(A \cap B)$ and $M(Q-P)=M\left(B^{\prime}-A\right)$, then we have

$$
\begin{aligned}
S(P, Q) & =F_{S}(M(P \cap Q), M(Q-P)) \\
& =F_{S}\left(M(A \cap B), M\left(B^{\prime}-A\right)\right), \\
S(P, Q) & <F_{S}(M(A \cap B), M(B-A))=S(A, B)
\end{aligned}
$$

and

$S(P, Q)>F_{S}\left(M\left(A \cap B^{\prime}\right), M\left(B^{\prime}-A\right)\right)=S\left(A, B^{\prime}\right)$.

Let us now prove that there exists such a pair $(P, Q)$. We can consider $P=A$ and $Q$ with a membership function $f_{Q}(x)$ equal to $f_{B}(x)$ for all $x$ such that $f_{B}(x) \leqslant f_{A}(x)$ and $f_{Q}\left(x^{\prime}\right) \geqslant f_{B}\left(x^{\prime}\right)$ for all $x^{\prime}$ such that $f_{B}\left(x^{\prime}\right) \geqslant f_{A}\left(x^{\prime}\right)$. We get $M(Q \cap P)=M(A \cap B)$ and 
we can choose $Q$ such that $M(Q-P)=M(Q-A)=$ $M\left(B^{\prime}-A\right)$ because of $\mathrm{D} 2$.

The solvability property means that if $B$ resembles $A$ more than $B^{\prime}$, there exist two objects $P$ and $Q$, with the same common information as $A$ and $B$, with the same difference as $B^{\prime}$ and $A$, providing a measure of resemblance in the interval $] S\left(A, B^{\prime}\right), S(A, B)[$.

This last property is, again, a property which can be linked to the notion of solvability introduced by Tversky.

Usually, $M$-measures of satisfiability are not symmetrical, which means that, for most of the fuzzy subsets $A$ and $B, S(A, B) \neq S(B, A)$, because $A$ is taken as a reference and, further, $S$ depends on $B-A$ and not on $A-B$. Let us remark that Tversky also states that measures of similarity must not be symmetrical.

Examples of $M$-measures of satisfiability are the following:

- $S(A, B)=1-\sup _{f_{A}(x)=0} f_{B}(x)=1-M_{2}\left(B-{ }_{2} A\right)$

[2] (Example 4) for normalized fuzzy sets, with $M_{2}$ and the difference defined by -2 .

$-S(A, B)=\inf _{x} \min \left(1-f_{B}(x)+f_{A}(x), 1\right)=1-$ $M_{2}(B-1 A)$ [4] (Example 5) for normalized fuzzy sets, with $M_{2}$ and the difference defined by $-{ }_{1}$.

- $S(A, B)=M(A \cap B) / M(B)=M(A \cap B) /(M(A \cap B)$ $+M(B-A)$ ) (Example 6), with the fuzzy set measure $M_{1}$ or $M_{3}$ and the difference defined by -1 .

\subsection{Measures of inclusion}

The second family of measures of similitude we consider takes into account the notion of inclusion. Many applications need the use of measures of inclusion, which consider again a reference object $A$ and looks at the characteristics of $B$ which are common to $A$.

Definition 3.3. An $M$-measure of inclusion $S$ on $\Omega$ is an $M$-measure of similitude such that

$\mathrm{AF} 2: F_{S}(0, v, w)=0$ whatever $v$ and $w$ may be,

AF5: $S$ is reflexive,

AF6: $F_{S}(u, v, w)$ is independent of $v$.

This definition is more flexible than most of the definitions of degree of inclusion in the literature $[1,19]$. If $B$ is included in $A$ with a degree $D(A, B)$, most of the degrees are based on fuzzy implications: $D(A, B)$ is null for all the descriptions $A$ and $B$ such that the support of $B$ (with Gödel implication for instance) or the kernel of $B$ (with Lukasiewicz implication for instance) is not included in the support of $A$, depending on the chosen implication. This constraint is very strong and does not enable us to evaluate the degree of inclusion of many descriptions $B$ which are reasonably comparable with $A$. We further assume that reflexivity is a necessary property of a degree of inclusion, since it evaluates to which extent the part of $B$ common to $A$ fills up $A$ and in the particular case where $B=A$, it reaches its maximum. Nevertheless, the reflexivity property is not always required (see Kleene-Dienes implication, for instance).

Proposition 3.5. An M-measure of inclusion satisfies the following property:

P6: Exclusiveness.

P14: Set-inclusion. If $M$ is such that $M(A)=$ $M(A \cap B)+M(A-B)$ for any $A$ and $B$, then any $M$-measure of inclusion is monotonous with respect to $M(A \cap B)$ and, as a consequence, it is monotonous with respect to $B$ for a fixed $A$ :

- if $M(A \cap B) \leqslant M\left(A \cap B^{\prime}\right)$ then $S(A, B) \leqslant S\left(A, B^{\prime}\right)$,

- if $B \subseteq B^{\prime}$ then $S(A, B) \leqslant S\left(A, B^{\prime}\right)$.

\section{Proof.}

- P6 is, as it was noticed for satisfiability measures, a direct consequence of AF2.

- Suppose that $M(A)=M(A \cap B)+M(A-B)=$ $M\left(A \cap B^{\prime}\right)+M\left(A-B^{\prime}\right)$ for every $B^{\prime}$; hence, if $M(A \cap B) \leqslant M\left(A \cap B^{\prime}\right)$, we obtain $M(A-B) \geqslant$ $M\left(A-B^{\prime}\right)$. Then, because of AF $1, S(A, B) \leqslant S\left(A, B^{\prime}\right)$. - Moreover, if $B \subseteq B^{\prime}$, we have also $M(A \cap B)$ $\leqslant M\left(A \cap B^{\prime}\right)$ and $M(A-B) \geqslant M\left(A-B^{\prime}\right)$ AF1 entails the same result as the previous one: $S(A, B)$ $\leqslant S\left(A, B^{\prime}\right)$

Properties of $M$-elementary internal and external information analogous to $\mathrm{P} 11$ and $\mathrm{P} 12$ are satisfied by $M$-measures of inclusion.

Examples of $M$-measures of inclusion are the following:

- $S(A, B)=|A \cap B| /|A|=M_{3}(A \cap B) /\left(M_{3}(A \cap B)+\right.$ $M_{3}(A-B)$ ) [21] (Example 10), with the fuzzy set measure $M_{3}$ and the difference defined by $-{ }_{1}$. 


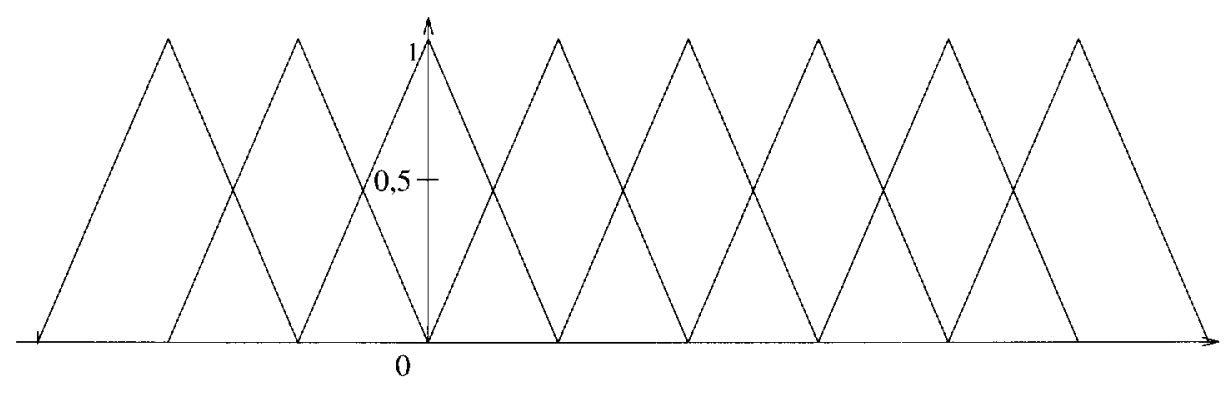

Fig. 2. Fuzzy partition in a fuzzy control.

$-S(A, B)=\inf _{x} \min \left(1-f_{A}(x)+f_{B}(x), 1\right)=1-$ $M_{2}\left(A-{ }_{1} B\right)[6]$ (Example 11) for normalized fuzzy sets.

The classical degree of inclusion (Zadeh's inclusion)

$$
\begin{aligned}
S(A, B) & =\frac{1}{|\Omega|} \sum_{x} \min \left(1,1-f_{A}(x)+f_{B}(x)\right) \\
& =1-\frac{1}{|\Omega|} M_{3}\left(A-{ }_{1} B\right)
\end{aligned}
$$

is an $M$-measure of comparison satisfying all the requirements of an $M$-measure of inclusion, except the fact that $S(A, B) \neq 0$ for $A$ and $B$ such that $A \cap B=\emptyset$.

\subsection{Measures of resemblance}

The last family of measures of similitude we consider takes into account two objects and looks at the characteristics they have in common, without regarding one of them as a reference.

Definition 3.4. An $M$-measure of resemblance on $\Omega$ is an $M$-measure of similitude $S$ which satisfies properties of

AF5: Reflexivity. $S(A, A)=1$.

AF7: Symmetry. $S(A, B)=S(B, A)$.

These properties have been pointed out by Kaufmann as describing resemblances [11].

$M$-measures of resemblance $S$ which satisfy an additional property of $T$-transitivity, for a t-norm $T$, $S(A, B) \geqslant T(S(A, C), S(C, B)), \forall A, B, C \in F(\Omega)$, are known as indistinguishability relations [26, 24]. In the case where $T$ is the minimum, we obtain well-known measures of similarity [28].

The property of symmetry of $M$-measures of resemblance entails that $F(u, v, w)$ is symmetrical in $v$ and $w$ :
$F(u, v, w)=F(u, w, v)$. In the particular case where all the descriptions are such that $M(A-B)=M(B-A)$, any $M$-measure of satisfiability $S$ is a measure of resemblance since it is already reflexive. Further, $S$ becomes symmetrical because of this special condition. It is, for instance, the case if we consider descriptions defined as classes of a fuzzy partition commonly used in fuzzy control with symmetrical shapes of membership functions, identical for all the classes located at regular intervals as indicated in Fig. 2.

Examples of $M$-measures of resemblance are the following:

- $S(A, B)=\exp \left(-\beta\left|d_{r}(A, B)\right|\right)[15]$ (Example 7) where $\beta>0$ and $d_{r}(A, B)=\left(\sum\left|f_{A}-f_{B}\right|^{r}\right)^{1 / r}$, for $r \geqslant 1$, the generalized geometric distance for fuzzy sets. This quantity is a product-transitive indistinguishability relation; hence it is an $M$-measure of resemblance, with the fuzzy set measure $M_{5}$ and the difference defined by -1 .

- $S(A, B)=M(A \cap B) / M(A \cup B)[6]$ (Example 8) for $M$ such that $M(A \cup B)=M(A \cap B)+M(A-B)$ $+M(B-A)$, for instance, $M_{1}$ or $M_{3}$ and with the difference defined by -1 .

$-S(A, B)=1-(1 /|\Omega|) \sum_{x}\left|f_{A}(x)-f_{B}(x)\right|=1-$ $(1 /|\Omega|)\left(M_{3}(A-B)+M_{3}(B-A)\right)[6]$ (Example 9) with the fuzzy set measure $M_{3}$ and the difference -1 .

\section{Measures of dissimilarity}

Many measures of comparison of fuzzy sets in the literature are based on a kind of distance between their membership functions $[12,16,13,11]$. We give the requirements necessary to their introduction in the general framework. 
Definition 4.1. An $M$-measure of dissimilarity $S$ on $\Omega$ is an $M$-measure of comparison satisfying the following requirements:

AF8: Minimality. $S(A, A)=0$.

AF9: $F_{S}(u, v, w)$ is independent of $u$ and increasing in $v$ and $w$.

Clearly, a distance is a measure of dissimilarity which is symmetrical and satisfies the triangular inequality.

Examples of $M$-measures of dissimilarity are the following:

$-S(A, B)=|A \square B|=(1 /|\Omega|)\left(\sum_{x} f_{A-B}(x)+\right.$ $\sum_{x} f_{B \rightarrow A}(x)$ ) (Example 12) where $A \square B$ defines the fuzzy subset of elements that approximately belong to $A$ and not to $B$ or conversely, is an $M$ measure of dissimilarity with the fuzzy set measure $M_{3}$ and the difference -1 .

- the normalized generalized geometric distance: $S(A, B)=\left((1 /|\Omega|) \sum\left|f_{A}-f_{B}\right|^{r}\right)^{1 / r}$, (Example 13) for $r \geqslant 1$, with the fuzzy set measure $M_{5}$ and the difference -1 .

$-S(A, B)=d_{\infty}(A, B)=\sup \left|f_{A}(x)-f_{B}(x)\right|[13]$ (Example 14), with the fuzzy set measure $M_{5}$ and the difference $-{ }_{1}$.

\section{Aggregation of measures of similitude}

The chosen approach consists in establishing a comparison attribute by attribute (thus, a measure of comparison can correspond to each pair of attributes). We obtain as many values of comparison as there exists attributes. The step of aggregation of those values is then necessary. This approach is not Tversky's approach. Indeed, he suggests to compare objects globally even if he considers all the features of an object. A measure of similarity, in Tversky's axiomatic, should take features of objects into account once for all. Thus, a comparison of objects entails one value: the step of aggregation does not exist.

We suppose that a fuzzy measure $M^{i}$ and a difference - are defined on every $\Omega_{i}, i \in[1, n]$. We consider an $M^{i}$-measure of similitude $S_{i}$ on $\Omega_{i}, i \in$ $[1, n]$.

Let $\Omega$ be a set of objects described by means of attributes defined on sets $\Omega_{1}, \Omega_{2}, \ldots, \Omega_{n}$. An object $O$ is associated with values $A_{1}, A_{2}, \ldots, A_{n}$ of the at- tributes, respectively, defined as fuzzy subsets of $\Omega_{1}, \Omega_{2}, \ldots, \Omega_{n}$. Let us consider another object $O^{\prime}$ associated with $B_{1}, B_{2}, \ldots, B_{n}$. We say that

- an object $O$ is included in an object $O^{\prime}$ if and only if $A_{i}$ is included in $B_{i}$ for every $i$,

- the intersection of $O$ and $O^{\prime}$ is defined as the object $O \cap O^{\prime}$ with values of attributes $A_{i} \cap B_{i}$,

- the difference between objects $O$ and $O^{\prime}$ is defined as the object $O-O^{\prime}$ with values of attributes $A_{i}-B_{i}$.

- the measure of information $\mathscr{M}$ is a mapping defined on $\Omega$ and lying in $\mathbb{R}^{+n}$ such that, for every $O$ in $\Omega$

$$
\mathscr{M}(O)=\left(M^{1}\left(A_{1}\right), M^{2}\left(A_{2}\right), \ldots, M^{n}\left(A_{n}\right)\right) .
$$

As a consequence, $\mathscr{M}$ is monotonous: if $O \subseteq O^{\prime}$ then $M^{i}\left(A_{i}\right) \leqslant M^{i}\left(B_{i}\right) \forall i \in[1, n]$ and we write $\mathscr{M}(O) \preceq$ $\mathscr{M}\left(O^{\prime}\right)$ with minimal element $\bigcirc=(0, \ldots, 0)$.

We need to give a general degree of similitude $S$ defined on the set of objects $\Omega$, satisfying properties analogous to those required from each $S_{i}$, since they are natural characterizations for the evaluation of resemblances [17]. We prove the following.

Proposition 5.1. Let $S_{i}$ be an $M^{i}$-measure of similitude on $\Omega_{i}$, for $1 \leqslant i \leqslant n$. For any triangular norm $T$, the measure defined for any pair $\left(O, O^{\prime}\right)$ of objects of $\Omega$ by $\left.S\left(O, O^{\prime}\right)=T\left(S_{1}\left(A_{1}, B_{1}\right), \ldots, S_{n}\left(A_{n}, B_{n}\right)\right)\right)$ satisfies axiom $A F 1$ and can be regarded as an $\mathscr{M}$ measure of similitude.

This property is obvious because of the monotonicity of triangular norms.

Let us now consider the OWA operators [27] defined by $W=\left\{w_{1}, \ldots, w_{n}\right\}$ such that $w_{i} \in[0,1] \forall i$, $\sum_{i=1}^{n} w_{i}=1$ and

$$
\begin{aligned}
h_{W}:[0,1]^{n} & \rightarrow[0,1], \\
h_{W}\left(x_{1}, \ldots, x_{n}\right) & =\sum_{i=1}^{i=n} w_{i} b_{i},
\end{aligned}
$$

where $b_{i}$ is the $i$ th largest value among $x_{1}, \ldots, x_{n}$. They can be used to aggregate measures of resemblance.

Proposition 5.2. Let $S_{i}$ be an $M^{i}$-measure of similitude on $\Omega_{i}$, for $1 \leqslant i \leqslant n$. For any $O W A$-operator $h_{W}$, the measure defined for any pair $\left(O, O^{\prime}\right)$ of 


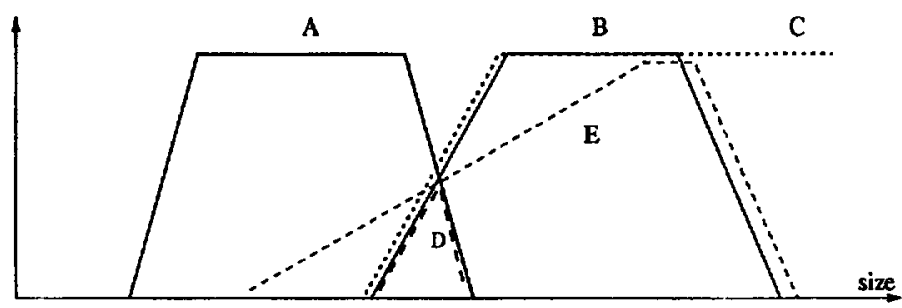

\begin{tabular}{|l|lll}
$M_{1}$ fuzzy measure & $S_{1}(A, C) \leq S_{1}(A, B)<1$ & $S_{1}(A, D)=1 \geq S_{1}(A, B)$ & $S_{1}(A, E) \geq S_{1}(A, B)$ \\
$S_{4}$ satisfiability & $S_{2}(A, C) \leq S_{2}(A, B)$ & $1 \geq S_{2}(A, D) \geq S_{2}(A, B)$ & $S_{2}(A, E) \geq S_{2}(A, B)$ \\
$S_{2}$ resemblance & $S_{3}(A, C)=S_{3}(A, B)$ & $S_{3}(A, D)=S_{3}(A, B)$ & $S_{3}(A, E) \geq S_{3}(A, B)$ \\
$S_{3}$ inclusion & $S_{4}(A, C) \geq S_{4}(A, B)$ & $S_{4}(A, D) \leq S_{4}(A, B)$ & $S_{4}(A, E) \leq S_{4}(A, B)$ \\
$S_{4}$ dissimilarity & & &
\end{tabular}

Fig. 3. The behaviour of different $M$-measures of comparison.

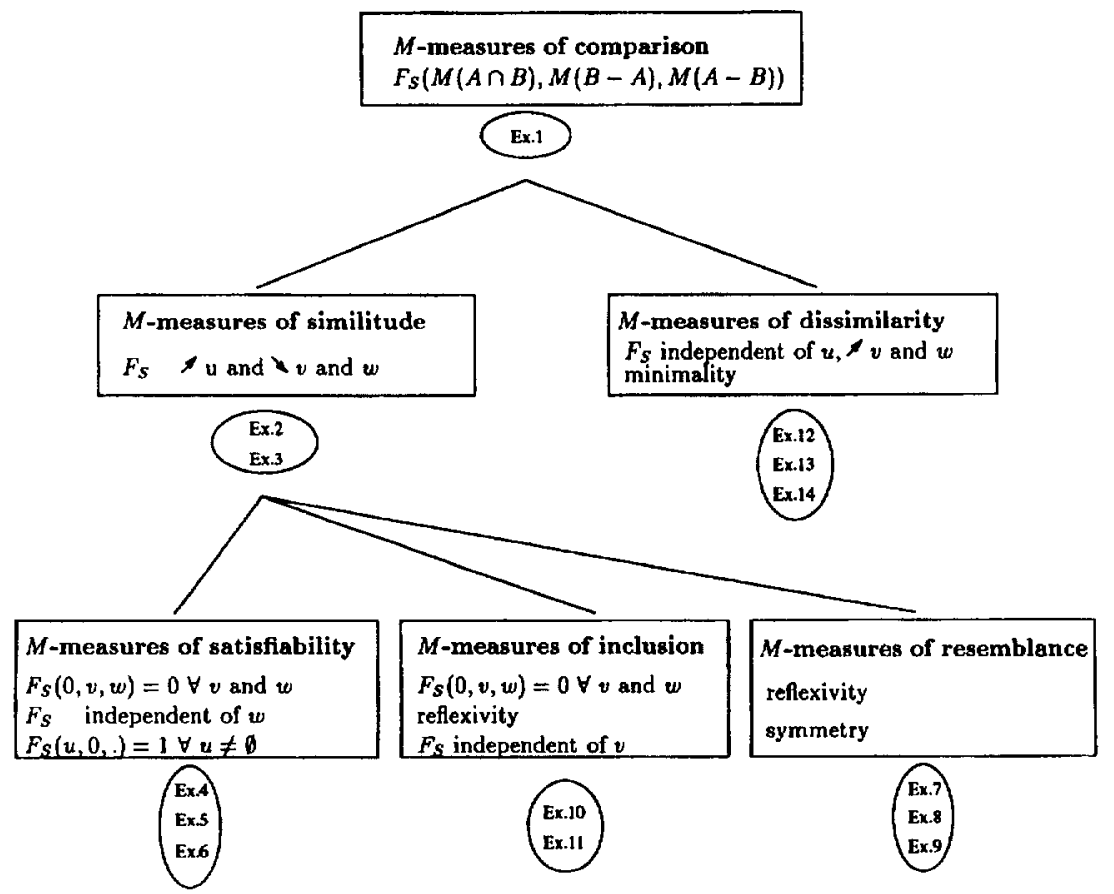

Fig. 4. Classification of $M$-measures of comparison.

objects of $\Omega$ by

$S\left(O, O^{\prime}\right)=\sum_{i=1}^{i=n} w_{i} b_{i}$

with $b_{i}$ the ith largest value of $S_{i}\left(A_{i}, B_{i}\right)$, satisfies axioms $A F 1$ and it can be regarded as an $\mathscr{M}$-measure of similitude.
This result follows from the monotonicity of OWA operators.

Proposition 5.3. Let $S_{i}$ be an $M^{i}$-measure of satisfiability on $\Omega_{i}$, for $1 \leqslant i \leqslant n$. The aggregation of $S_{i}$ for $1 \leqslant i \leqslant n$ by means of a triangular norm or an OWA-operator provides measures satisfying axioms 
$A F 2, A F 3$ and $A F 4$, which can be regarded as $\mathscr{M}$ measures of satisfiability.

The same kind of study can be extended to measures of resemblance, inclusion and dissimilarity.

\section{Conclusion}

We can see in Fig. 3 the differences existing among $M$-measures of comparison because of their multiple aspects.

The classification we propose (Fig. 4) covers most of degrees of comparison of fuzzy sets used in the literature. Some of them are excluded from this framework, because they have properties which do not seem natural, from our point of view.

New quantities can be easily deduced by replacing the difference -1 by another one, for instance -2 , and the currently used fuzzy measure by another one, already proposed or not.

Many extensions or utilizations of our work can be studied. For instance, properties of similarity together with dissimilarity can be used to obtain interval-valued comparison measures. Applications in database retrieval, analogical reasoning, case-based reasoning sytems or image processing prove that some measures of comparison must be preferred to other ones for reasons specific to the field of utilization.

\section{References}

[1] W. Bandier and L. Kohout, Fuzzy power sets and fuzzy implication operators, Fuzzy Sets and Systems 4 (1980) 13-30.

[2] B. Bouchon-Meunier, Fuzzy similitude and approximate reasoning, in: P.P. Wang, Ed., Advances in Fuzzy Theory and Technology (Bookwrights Press, 1993) 161-166.

[3] B. Bouchon-Meunier and M. Rifqi, Resemblance in database utilization, Proc. 6th IFSA World Congress, Sao Paulo, 1995.

[4] B. Bouchon-Meunier and L. Valverde, Analogy relations and inference, Proc. 2nd IEEE Internat. Conf. on Fuzzy Systems, San Francisco (1993) 1140-1144.

[5] A. De Luca and S. Termini, A definition of a non-probability entropy in the setting of fuzzy sets theory, Inform. and Control 20 (1972) 301-312
[6] D. Dubois and H. Prade, Fuzzy Sets and Systems, Theory and Applications (Academic Press, New York, 1980).

[7] D. Dubois and H. Prade, Fuzzy set-theoretic differences and inclusions and their use in fuzzy arithmetics and analysis, Proc. 5th Internat. Seminar on Fuzzy Set Theory, Linz, Austria, 1983.

[8] D. Dubois and H. Prade, Ranking fuzzy numbers in the setting of possibility theory, Inform. Sci. 30 (1983) 183-224.

[9] F. Esteva, P. García, L. Godó, E. Ruspini and L. Valverde, On similarity logic and the generalized modus ponens, Proc. FUZZY-IEEE'94, Orlando, 1994.

[10] J. Gasos and A.L. Ralescu, The direct and inverse problem of the matching of fuzzy sets depending on fuzziness, Kybernetes 24 (1995) 9-17.

[11] A. Kaufmann, Introduction à la théorie des sous-ensembles flous, Vol. 3 (Masson, Paris, 1973).

[12] L.T. Kóczy and K. Hirota, Ordering, distance and closeness of fuzzy sets, Fuzzy Sets and Systems 59 (1993) 281-293.

[13] M. Nowakowska, Methodological problems of measurement of fuzzy concepts in the social sciences, Behavioral Sci. 22 (1977) 107-115.

[14] M.-N. Omri, Système interactif flou d'aide à l'utilisation de dispositifs techniques: le système SIFADE, Ph.D. Thesis, Université Pierre et Marie Curie, Paris, France, 1994.

[15] S.V. Ovchinnikov, Representations of transitive fuzzy relations, in: H.J. Skala, S. Termini and E. Trillas, Eds., Aspects of Vagueness (Reidel, Dordrecht, 1984) 105-118.

[16] A.L. Ralescu and D.A. Ralescu, Probability and fuzziness, Inform. Sci. 34 (1984) 85-92.

[17] M. Rifqi, Measures de similitude et leur agrégation, in: Rencontres logique floue et applications, LFA'95, Paris, 1995.

[18] D.W. Roberts, An anticommutative difference operator for fuzzy sets and relations, Fuzzy Sets and Systems 21 (1987) $35-42$.

[19] J-P Rossazza, Utilisation de hiérarchie de classes floues pour la représentation des connaissances imprécises et sujettes à exception: le système SORCIER, Ph.D. Thesis, Université Paul Sabatier, Toulouse, France, 1990.

[20] E.H. Ruspini, On the semantics of fuzzy logic, Internat. $J$. Approximate Reasoning 5 (1991) 45-88.

[21] E. Sanchez, Inverses of fuzzy relations, applications to possibility distributions and medical diagnosis, Fuzzy Sets and Systems 2 (1979) 75-86.

[22] M. Sugeno, Theory of fuzzy integral and its application, Ph.D Thesis, Tokyo Institute of Technology, Japan, 1974.

[23] P. Suppes, D.H. Krantz, R. Duncan Luce and A. Tversky, Foundations of Measurement, Vol. 2 (Academic Press, New York, 1990) 159-222.

[24] E. Trillas and L. Valverde, On implication and indistinguishability in the setting of fuzzy logic, in: J. Kacprzyk and R.R. Yager, Eds., Management Decision Support Systems Using Fuzzy Sets and Possibility Theory (Verlag TUV, Rheinland, 1984).

[25] A. Tversky, Features of similarity, Psycho. Rev. 84 (1977) $327-352$. 
[26] L. Valverde, On the structure of $t$-indistinguishability operators, Fuzzy Sets and Systems 17 (1985) 313-328.

[27] R.R. Yager, On ordered weighted averaging operators on multicriteria decision-making, IEEE Trans. Systems Man Cybernet. 18 (1988) 183-190.

[28] L.A. Zadeh, Similarity relations and fuzzy orderings, Inform. Sci. 3 (1971) 177-200.
[29] L.A. Zadeh, The concept of a linguistic variable and its application to approximate reasoning, Inform. Sci. 8 and 9 (1975) 199-249, 301-357, 43-80.

[30] R. Zwick, E. Carlstein and D.V. Budescu, Measures of similarity among fuzzy concepts: a comparative analysis, Internat. J. Approximate Reasoning 1 (1987) 221-242. 